\title{
Isotemporal substitution of sedentary time with physical activity and its associations with frailty status
}

This article was published in the following Dove Press journal:

Clinical Interventions in Aging

\author{
Koutatsu Nagai' \\ Kayoko Tamaki ${ }^{2}$ \\ Hiroshi Kusunoki \\ Yosuke Wada ${ }^{3}$ \\ Shotaro Tsuji ${ }^{4}$ \\ Masako Ito ${ }^{5}$ \\ Kyoko Sano 5 \\ Manabu Amano ${ }^{6}$ \\ Soji Shimomura ${ }^{7}$ \\ Ken Shinmura ${ }^{2}$ \\ 'Department of Physical Therapy, \\ School of Rehabilitation, Hyogo \\ University of Health Sciences, Kobe, \\ Japan; ${ }^{2}$ Department of General \\ Medicine, Hyogo College of Medicine, \\ Nishinomiya, Japan; ${ }^{3}$ Department \\ of Rehabilitation, Hyogo College of \\ Medicine Sasayama Medical Center, \\ Sasayama, Japan; ${ }^{4}$ Department of \\ Orthopaedic Surgery, Hyogo College \\ of Medicine Sasayama Medical Center, \\ Sasayama, Japan; ${ }^{5}$ Department of \\ Occupational Therapy, School of \\ Rehabilitation, Hyogo University of \\ Health Sciences, Kobe, Japan; ${ }^{6}$ School \\ of Pharmacy, Hyogo University \\ of Health Sciences, Kobe, Japan; \\ ${ }^{7}$ Department of General Medicine and \\ Community Health Science, Hyogo \\ College of Medicine Sasayama Medical \\ Center, Sasayama, Japan
}

Correspondence: Koutatsu Nagai Department of Physical Therapy, School of Rehabilitation, Hyogo University of Health Sciences, I-3-6 Minatojima, Chuo-ku, Kobe, Hyogo 650-8530, Japan

Tel +8I 783043047

Fax +8I 783042747

Email nagai-k@huhs.ac.jp
Objectives: Recently, isotemporal substitution has been developed to substitute activity time for an equivalent amount of another activity. This study employed this method to demonstrate the effects of replacing sedentary behavior (SB) time with an equivalent amount of light-intensity physical activity (LPA) and moderate-to-vigorous intensity physical activity (MVPA) on the risk for different severities of frailty.

Methods: A total of 886 older adults (average age 73.6 years, female $70 \%$ ) participated in this cross-sectional study. Frailty status was assessed according to the cardiovascular health study criteria.

Main outcome measures: Wrist-worn accelerometers were used to measure SB, LPA, and MVPA. Isotemporal substitution models were applied to show the estimated effects of substituting 30 min of SB with an equal amount of time spent in LPA or MVPA on the risk for pre-frailty and frailty.

Results: The physical activity level and SB were not associated with the incidence of pre-frailty. However, a 16\% (OR: 0.84; 95\% CI: 0.78-0.90) and 42\% (OR: 0.58; 95\% CI: 0.37-0.92) decrease in frailty risk was noted when SB was substituted with LPA and MVPA, respectively, in the crude model. In the adjusted model, the significant effect was sustained for LPA (OR: 0.86; 95\% CI: 0.80-0.92) but not for MVPA (OR: 0.74; 95\% CI: 0.47-1.17).

Conclusions: This study indicates that replacing $30 \mathrm{~min}$ of $\mathrm{SB}$ with an equivalent amount of LPA decreases the risk for frailty in older adults. Moreover, increasing LPA seems more feasible than increasing MVPA in older adults, with substantial benefit.

Keywords: isotemporal substitution, frailty, physical activity, sedentary behavior

\section{Introduction}

Physical frailty, a syndrome related to health problems such as falls and activity limitation, as well as hospitalization, increases the risk of early mortality and medical costs. ${ }^{1,2}$ Thus, focusing on the prevention and treatment of frailty syndrome in older adults is crucial and interventions aimed at improving the symptoms of frailty should be beneficial for the patient and society. ${ }^{3}$

Increased physical activity (PA) is a key factor in the prevention of physical deterioration, including frailty. ${ }^{4} \mathrm{PA}$ guidelines recommend that older adults engage in a minimum of 150 min per week of moderate-to-vigorous intensity PA (MVPA) ${ }^{5}$ to maintain a healthy physical condition. However, few older adults follow the current PA guideline,${ }^{6}$ and focusing on the increment of MVPA may be infeasible, especially for a high-risk population such as older adults with frailty. ${ }^{7}$ 
Poor health outcomes such as cardiovascular disease, cancer, impaired metabolism, and mortality are associated with sedentary behaviors (SBs) including watching television, desk work, and driving, independent of MVPA., ${ }^{8,9}$ Moreover, recent studies have demonstrated that lightintensity PA (LPA) could improve the physical and cognitive health of older adults. ${ }^{7,10}$ Older adults with frailty symptoms show prolonged SB and decreased LPA compared to healthier populations. ${ }^{11}$ Decreasing SB and increasing LPA as a health promotion strategy may be more acceptable for these inactive populations. Yet, to date, no study has examined whether replacing SB with LPA (or MVPA) reduces the risk for different severities of frailty.

Recently, a new approach, the isotemporal substitution, has been developed to substitute activity time for an equivalent amount of another activity. ${ }^{12}$ Using this method should enable the investigation of the effect of replacing SB with LPA and MVPA on frailty status in older adults. Therefore, this study aimed to ascertain the effects of replacing SB with an equivalent amount of PA (LPA and MVPA) on the risk for different severities of frailty.

\section{Methods}

\section{Study design and participants}

This study employed a cross-sectional study design. The data were collected from August 2015 to December 2017. Community-dwelling older adults were recruited via advertisement in the community newspaper or oral announcement by medical staff. Participants received oral and written explanations concerning the study and provided their written informed consent prior to participating in the study. The inclusion criteria were an age of 65 years or older and the ability to walk independently (or using a cane). The exclusion criteria were neurological impairment (eg, stroke, Parkinson's disease, or paresis of the lower limbs), cognitive impairment (Mini Mental State Examination [MMSE] score of $<23),{ }^{13}$ and participants with missing data values. Neurological impairment and cognitive impairment were set as the criteria because of conflicting evidence for the validity and reliability of accelerometer measurements, ${ }^{14}$ and concern about the reliability of the questionnaire regarding frailty, respectively. Participants with osteoarthritis or rheumatoid arthritis who had walking difficulty were not excluded. This study involved 963 community-dwelling older adults. Of these, 77 participants were excluded, and the remaining 886 participants (average age 73.6 years, female $70 \%$ ) were included in the analysis.

\section{Assessment of physical frailty and covariates}

Physical frailty was characterized by limitations in at least three of the following conditions based on the cardiovascular health study criteria: ${ }^{1,15}$ slow gait speed, weakness, exhaustion, low activity, and weight loss. The number of corresponding components was used as the frailty score. Participants who did not exhibit any of the components were considered non-frail and those who showed one or two components were considered pre-frail. To calculate walking speed, which was used as the stratification variable, participants were asked to walk down a $12-\mathrm{m}$ walkway at their preferred speed and the time taken to reach $10 \mathrm{~m}$ was measured. ${ }^{16}$ Slow gait was established according to a cutoff point of $<1.0 \mathrm{~m} / \mathrm{s} .{ }^{1}$ Weakness was assessed using maximum grip strength, which was measured in kilograms (kg) using a Smedley-type handheld dynamometer (GRIP-A; Takei Ltd, Niigata, Japan). Weakness was established according to sex-specific cutoff points ( $<26 \mathrm{~kg}$ for men, $<18 \mathrm{~kg}$ for women). ${ }^{17}$ Exhaustion was considered present if participants responded with "yes" to the following question from the Kihon Checklist developed by the Japanese Ministry of Health, Labor, and Welfare: 18 "In the last 2 weeks, have you felt tired without a reason?" The role of PA was assessed via the question "Do you engage in physical exercise?" If participants answered "no" we considered their PA level to be low. Weight loss was assessed with the question "Have you lost $2 \mathrm{~kg}$ or more in the past 6 months?" 18 We also collected demographic data, including age, sex, and medical history, which were included as covariates.

\section{Physical activity and sedentary behavior measurement}

PA and SB were measured for 14 consecutive days using a wrist-worn accelerometer (Actiband, TDK Co., Tokyo, Japan). The correlation between wrist- and hip-worn accelerometers has been reported as moderate $(r=0.55-0.86),{ }^{19}$ and wrist-worn accelerometers could classify sedentary and other activities with high accuracy. ${ }^{20}$ The epoch duration for the accelerometer was $5 \mathrm{~min}$. Participants were instructed to wear the accelerometer constantly, including during sleep; however, they could remove it during bathing if they desired. Participants did not receive feedback regarding PA during the measurement. Exercise intensity was classified according to accelerometer data, as follows: sedentary, $<1.5$ metabolic equivalents (METs); LPA, $\geq 1.5-<3$ METs; and MVPA, $\geq 3$ METs. ${ }^{21}$ The average sedentary time and PA levels were determined for each 
intensity level. Thereafter, a period of self-reported sleeping time was excluded from the sedentary time. The data with continuous zero counts of PA of at least 600 min during the daytime were excluded as non-wear periods. ${ }^{22}$ Data for participants who provided complete data of at least 4 days of 10 or more hours/day of wear time were included in the analysis. ${ }^{11}$ The validity and reliability of the device have been reported in a previous study. ${ }^{23}$

\section{Statistics}

We applied one-way ANOVA to compare PA and SB between the groups according to frailty status. The pointbiserial correlation coefficient was used to investigate the correlation between PA and each frailty subdomain. Multiple logistic regression models were used to examine associations between exposure ( $30 \mathrm{~min}$ of sedentary time and different PA intensity levels) and outcomes (incidence risk of pre-frailty and frailty). The first analyses were single-factor, examining the association of each intensity category (SB, LPA, and MVPA) with frailty status, with adjustment for only wear time (model 1), and additional adjustment for covariates (age, gender, and education) (model 2). The second analyses involved partition models examining the association of each intensity category while controlling for each of the other categories of activity, with adjustment for only wear time (model 3), and additional adjustment for covariates (model 4). The third analyses involved isotemporal substitution models that represented the estimated effects of substituting SB with an equal amount of time spent in LPA or MVPA, without (model 5) and with (model 6) adjustment for covariates. ${ }^{12}$ In this model, SB was excluded, whereas, total wear time was kept constant in the equation. For isotemporal substitution modeling, the outcome can either be continuous or dichotomous. ${ }^{24}$ Data were analyzed using IBM SPSS ver. 24 (IBM Corporation, Armonk, NY, USA). Statistical significance was set at $P<0.05$.

\section{Results}

Table 1 shows the general characteristics of the participants. Fifty participants (5.6\%) were classified as frail (Table 2). The average daily accelerometer wear time, excluding sleep time was 1,015 min, of which $510 \mathrm{~min}(50 \%), 463 \mathrm{~min}(46 \%)$, and $42 \mathrm{~min}(4 \%)$ were spent in SB, LPA, and MVPA, respectively (Table 2). Compared to the other groups, SB time was significantly higher, and LPA and MVPA were significantly lower in the frailty group $(P<0.001)$, although no significant difference regarding these parameters was noted between the
Table I Characteristics of participants $(n=886)$

\begin{tabular}{ll}
\hline Characteristic & Value \\
\hline Age, years, mean (SD) & $73.6(7.0)$ \\
Height, cm, mean (SD) & $154.9(8.1)$ \\
Body mass index, kg/m², mean (SD) & $22.7(3.08)$ \\
Female sex, $\mathrm{n}(\%)$ & $619(70)$ \\
Medication, $\mathrm{n}$, median (IQR) & $\mathrm{I}(3)$ \\
Comorbidity & \\
Hypertension, $\mathrm{n}(\%)$ & $394(45)$ \\
Diabetes, $\mathrm{n}(\%)$ & $1 \mathrm{I}(\mathrm{I})$ \\
Kidney disease, $\mathrm{n}(\%)$ & $24(3)$ \\
Cardiovascular disease, $\mathrm{n}(\%)$ & $73(8)$ \\
Osteoporosis, $\mathrm{n}(\%)$ & $99(\mathrm{II})$ \\
MMSE, median (IQR) & $29(4)$ \\
Education, years, median (IQR) & $12(2)$ \\
Sleep time, h, mean (SD) & $6.7(\mathrm{I} . \mathrm{I})$ \\
Frailty status & \\
Frailty, $\mathrm{n}(\%)$ & $50(6)$ \\
Pre-frailty, $\mathrm{n}(\%)$ & $477(54)$ \\
Robust, $\mathrm{n}(\%)$ & $359(40)$ \\
Gait speed, m/s, mean (SD) & $1.4(0.3)$ \\
Grip strength, kg, mean (SD) & $26.7(7.6)$ \\
\hline
\end{tabular}

Abbreviations: IQR, interquartile range; MMSE, Mini Mental State Examination.

non-frail and pre-frailty group $(P=0.410)$. In the pre-frailty group, few people experienced a decline in physical function according to the frailty criteria slow gait speed (6\%) and weakness (10\%). According to the correlation analysis, slow gait speed and weakness were significantly related to all of PA the levels (Table 3). Low activity as a frailty criterion indicated low MVPA, but not LPA.

Table 4 shows the results of the single, partition, and isotemporal substitution models for the effects of SB, LPA, and MVPA on frailty according to the logistic regression analysis to predict pre-frailty and frailty. The PA level and SB were not associated with the incidence of pre-frailty in any of the models. In the single models regarding the prediction of frailty, every 30 -min increment of sedentary time was associated with a significantly increased OR for frailty even after adjustment for covariates. Increased LPA and MVPA were associated with a significantly decreased OR for frailty after adjustment. In the partition model, LPA and MVPA demonstrated significantly increased ORs in the crude model but no significance was found in the adjusted model. In the isotemporal substitution model, which substituted sedentary time (30 min) with an equal amount of LPA and MVPA time, a $16 \%$ (OR: 0.84 ; $95 \%$ CI: $0.78-0.90)$ and $42 \%$ (OR: 0.58 ; 95\% CI: $0.37-0.92$ ) decrease in the risk for frailty was noted for LPA and MVPA, respectively, in the crude model. In the adjusted model, the significant effect was sustained for LPA (OR: 0.86; 95\% CI: 0.80-0.92), but not for MVPA (OR: $0.74 ; 95 \%$ CI: $0.47-1.17$ ). 
Table 2 Physical activity and frailty subdomain in older adults $(n=886)$

\begin{tabular}{|c|c|c|c|c|c|}
\hline & Overall $(n=886)$ & Robust (n=359) & Pre-frailty $(n=477)$ & Frailty $(n=50)$ & $P$-value ${ }^{\text {a }}$ \\
\hline \multicolumn{6}{|l|}{ Physical activity, min/day, mean (SD) } \\
\hline Sedentary behavior & $510(170)$ & $488(153)$ & $508(169)$ & $683(197)$ & $<0.00 \mathrm{I}^{\mathrm{b}, \mathrm{c}}$ \\
\hline Light intensity & $463(150)$ & $478(136)$ & $468(149)$ & $310(170)$ & $<0.00 \mathrm{I}^{\mathrm{b}, \mathrm{c}}$ \\
\hline Moderate to vigorous intensity & $42(34)$ & $45(3 \mathrm{I})$ & $4 \mid(34)$ & $21(37)$ & $<0.00 \mathrm{I}^{\mathrm{b}, \mathrm{c}}$ \\
\hline Wear time & $1,015(74)$ & I,0II (74) & $\mathrm{I}, 017(75)$ & $1,013(67)$ & 0.41 \\
\hline \multicolumn{6}{|l|}{ Frailty criteria, n (\%) } \\
\hline Slow gait speed & $55(6.2)$ & $0(0)$ & $27(6)$ & $28(56)$ & - \\
\hline Weakness & $77(8.7)$ & $0(0)$ & $47(10)$ & $30(60)$ & - \\
\hline Exhaustion & $202(23)$ & $0(0)$ & $159(33)$ & $43(86)$ & - \\
\hline Low activity & $299(34)$ & $0(0)$ & $264(55)$ & $35(70)$ & - \\
\hline Weight loss & $133(15)$ & $0(0)$ & $108(23)$ & $25(50)$ & - \\
\hline
\end{tabular}

Notes: ${ }^{a}$ One-way ANOVA was used to compare each time between the groups. ${ }^{b}$ Significant difference according to post-hoc analysis between the robust and frailty groups $(P<0.05)$. 'Significant difference according to post-hoc analysis between the pre-frailty and frailty groups $(P<0.05)$.

\section{Discussion}

The present study showed that replacing 30 min of SB with an equivalent amount of LPA decreased the risk for frailty in older adults. Although MVPA seemed to significantly reduce the risk for frailty with a larger effect size (represented by the OR), the effect was not statistically significant. Notably, the PA level and SB did not predict the risk for pre-frailty.

Some studies have demonstrated that even light-intensity, and not moderate-to-vigorous, activity had a positive effect on health in older adults. A review by Powell et al found that health benefits begin with any increase above the very lowest levels of activity, and the greatest health and functional benefits are found for increment in activity within achieving the mainstream moderate-intensity prescription. ${ }^{25} \mathrm{~A}$ recent systematic review has demonstrated the effectiveness of lowintensity exercise (1.5-3.0 METs) on physical outcomes such as lower limb muscle strength, balancing, and flexibility. ${ }^{7}$ We also previously reported the feasibility of LPA increment compared to MVPA increment as an intervention and observed a positive effect of LPA on physical outcomes in older adults with frailty symptoms. ${ }^{23}$ Considering the positive effect of LPA in the present study, substituting SB with

Table 3 Correlations between physical activity and frailty subdomains in older adults $(n=886)$

\begin{tabular}{llll}
\hline Frailty subdomain & SB & LPA & MVPA \\
\hline Slow gait speed & $0.29^{\mathrm{a}}$ & $-0.30^{\mathrm{a}}$ & $-0.17^{\mathrm{a}}$ \\
Weakness & $0.14^{\mathrm{a}}$ & $-0.16^{\mathrm{a}}$ & $-0.12^{\mathrm{a}}$ \\
Exhaustion & $0.13^{\mathrm{a}}$ & $-0.12^{\mathrm{a}}$ & -0.00 \\
Low activity & 0.03 & 0.02 & $-0.13^{\mathrm{a}}$ \\
Weight loss & 0.04 & -0.02 & -0.01 \\
\hline
\end{tabular}

Notes: Point-biserial correlation coefficient was used to show the correlation between physical activity and frailty subdomains. ${ }^{a} P<0.0$ I.

Abbreviations: LPA, light-intensity physical activity; MVPA, moderate-to-vigorous physical activity; SB, sedentary behavior.
LPA seems an effective and feasible method for decreasing frailty risk.

In the current study, substituting SB with MVPA did not significantly predict frailty risk after adjustment for covariates. The suggested target PA for older adults is 150 min a week of MVPA. ${ }^{26}$ However, achieving this is challenging. A recent UK study reported that only $10 \%-15 \%$ of free-living older adults met the minimum standard for "sufficient activity" (>150 min/week of MVPA). ${ }^{6}$ Sparling et al suggested that the target was too high for many older adults. ${ }^{27}$ The proportion of the SD of MVPA was larger than that of LPA in the present study, which means MVPA includes larger individual variability compared to LPA. The fact that the statistical significance was not retained in the adjusted isotemporal substitution model suggests that MVPA is easily influenced by covariance. Accordingly, increasing MVPA seems a reasonable strategy for improving frailty status; however, it may not be as beneficial as LPA for all older adults. Nevertheless, the effect size of MVPA for decreasing the risk for frailty was large; thus, older adults who have potential to increase their MVPA should be encouraged to do so.

PA did not decrease significantly in pre-frail older adults compared to the non-frail and accordingly, the PA level did not predict pre-frailty as against the results of frailty in the present study. A previous study also showed that PA in older adults who are physically vulnerable and may be considered pre-frail was almost the same as that in non-frail older adults. ${ }^{11}$ Alternatively, 55\% of participants with pre-frailty fell into the "low activity" category, as a frailty criterion, and significant correlation between the criterion and actual PA measured by an accelerometer was found only in MVPA. Because PA assessment as a frailty criterion was evaluated by a simple questionnaire with the question "Do you engage 
Table 4 Single-factor, partition, and isotemporal substitution models for frailty in older adults ( $\mathrm{n}=886)$

\begin{tabular}{|c|c|c|c|c|c|c|}
\hline \multirow[t]{2}{*}{ Model } & \multicolumn{3}{|c|}{ OR for pre-frailty ${ }^{a}$} & \multicolumn{3}{|l|}{ OR for frailty } \\
\hline & SB & LPA & MVPA & SB & LPA & MVPA \\
\hline \multicolumn{7}{|l|}{ Single factor } \\
\hline \multirow[t]{3}{*}{ Crude model (model I) } & $1.02(1.00-1.05)$ & - & - & $1.23(1.16-1.30)^{b}$ & - & - \\
\hline & - & $0.99(0.96-1.01)$ & - & - & $0.8 \mathrm{I}(0.76-0.86)^{\mathrm{b}}$ & - \\
\hline & - & - & $0.91(0.80-1.02)$ & - & - & $0.34(0.21-0.55)^{b}$ \\
\hline \multirow[t]{3}{*}{ Adjusted model (model 2) } & $1.03(1.00-1.06)$ & - & - & $1.18(1.11-1.26)^{b}$ & - & - \\
\hline & - & $0.99(0.96-1.02)$ & - & - & $0.84(0.79-0.90)^{\mathrm{b}}$ & - \\
\hline & - & - & $0.89(0.78-1.01)$ & - & - & $0.5 \mathrm{I}(0.32-0.8 \mathrm{I})^{\mathrm{b}}$ \\
\hline \multicolumn{7}{|l|}{ Partition } \\
\hline Crude model (model 3) & I.04 (0.99-I.II) & $1.03(0.97-1.10)$ & $0.95(0.83-1.10)$ & $1.04(0.93-1.16)$ & $0.87(0.77-0.99)^{\mathrm{b}}$ & $0.60(0.38-0.96)^{b}$ \\
\hline Adjusted model (model 4) & $\mathrm{I} .05(0.99-1 . \mathrm{II})$ & $1.04(0.98-1.11)$ & $0.94(0.8 \mathrm{I}-1.09)$ & $1.04(0.93-1.17)$ & $0.89(0.78-1.02)$ & $0.77(0.48-1.23)$ \\
\hline \multicolumn{7}{|c|}{ Isotemporal substitution } \\
\hline Crude model (model 5) & Dropped & $0.98(0.96-1.02)$ & $0.91(0.80-1.04)$ & Dropped & $0.84(0.78-0.90)^{b}$ & $0.58(0.37-0.92)^{b}$ \\
\hline Adjusted model (model 6) & Dropped & $0.99(0.96-1.02)$ & $0.89(0.78-1.02)$ & Dropped & $0.86(0.80-0.92)^{\mathrm{b}}$ & $0.74(0.47-1.17)$ \\
\hline
\end{tabular}

Notes: ORs were calculated according to 30-min periods of sedentary time and PA intensity levels. In the model for pre-frailty, frail older adults were excluded. Age, gender, and education were included as covariates in the adjusted models. In the isotemporal substitution models, the OR represents the estimated effects of substituting sedentary time with an equal amount of time spent in LPA or MVPA. ${ }^{a}$ Older adults with frailty $(n=50)$ were excluded from the analysis time with an equal amount of time spent in LPA or MVPA. ${ }^{b} P<0.05$

Abbreviations: LPA, light-intensity physical activity; MVPA, moderate-to-vigorous physical activity; PA, physical activity; SB, sedentary behavior.

in physical exercise?", it might not substantially reflect the actual total PA including LPA or SB, which accounts for the vast majority of PA in daily life. Moreover, the reason why PA declined because of frailty and not pre-frailty may be explained by the presence or absence of a functional decline in older adults in each category. More than half of the older adults with frailty corresponded to the "slow gait speed" and "weakness" frailty criteria, which indicates that these people had apparent functional decline. However, only $6 \%$ and $10 \%$ of pre-frail participants corresponded to the "slow gait speed" and "weakness" criteria, respectively. Willey et al suggested that physical inactivity is related to slow gait speed in older adults. ${ }^{28}$ The present results also showed a correlation between functional decline, such as slow gait speed and weakness, and PA. The change in PA would be more sensitive in older adults with frailty who have a functional decline.

This study has several limitations. First, because of its cross-sectional study design, the ability to make causal inferences is limited. In addition, the isotemporal substitution model is a mathematical method to replace one behavior with another; thus, the results should be interpreted carefully. Second, although the accelerometer has some advantages compared to the questionnaire and self-reported methods, ${ }^{29}$ wrist-worn accelerometers cannot precisely detect differences between SB and standing activities. Moreover, this study employed a self-reported sleep time in order to omit sleep time from SB; thus, the measured time for PA and SB in the current study may include some errors. Nevertheless, a longer wear time exceeding 1,000 min, except for sleep, would be advantageous for estimating all PAs in the participants.

\section{Conclusions}

This study indicates that replacing 30 min of SB with an equivalent amount of LPA decreases the risk for frailty in older adults. However, replacing SB with any PA does not predict the risk for pre-frailty. In older adults, increasing LPA seems more feasible than increasing MVPA, with substantial benefit. Further studies with a longitudinal design are required to clarify the effects of SB relocation in older adults with different frailty severities.

\section{Ethics approval}

This study was approved by the ethics review board at Hyogo College of Medicine (No 201705-095) in accordance with the Declaration of Helsinki.

\section{Acknowledgement}

This work was supported by JSPS KAKENHI [Grant number: JP16KT0012] and Mitsui Life Social Welfare Foundation.

\section{Author contributions}

Koutatsu Nagai, Kayoko Tamaki, Hiroshi Kusunoki, and Ken Shinmura declare that they participated in the research design, data collection, analysis, and writing of this manuscript and that they have seen and approved the final version. Yosuke Wada, Shotaro Tsuji, Masako Ito, Kyoko Sano, Manabu Amano, and Soji Shimomura declare that they participated in the data collection and that they have seen and approved the final version. All authors contributed to data analysis, drafting and revising the article, gave final approval of the version to be published, and agree to be accountable for all aspects of the work. 


\section{Disclosure}

The authors report no conflicts of interest in this work.

\section{References}

1. Fried LP, Tangen CM, Walston J, et al. Frailty in older adults: evidence for a phenotype. J Gerontol A Biol Sci Med Sci. 2001;56(3):M146-M157.

2. Morley JE, Vellas B, van Kan GA, et al. Frailty consensus: a call to action. J Am Med Dir Assoc. 2013;14(6):392-397.

3. Theou O, Stathokostas L, Roland KP, et al. The effectiveness of exercise interventions for the management of frailty: a systematic review. J Aging Res. 2011;2011:1-19.

4. Savela SL, Koistinen P, Stenholm S, et al. Leisure-time physical activity in midlife is related to old age frailty. J Gerontol A Biol Sci Med Sci. 2013;68(11):1433-1438.

5. Organization WH. Global recommendations on physical activity for health. Geneva: World Health Organization; 2010.

6. Jefferis BJ, Sartini C, Lee IM, et al. Adherence to physical activity guidelines in older adults, using objectively measured physical activity in a population-based study. BMC Public Health. 2014;14:382.

7. Tse AC, Wong TW, Lee PH. Effect of low-intensity exercise on physical and cognitive health in older adults: a systematic review. Sports Med Open. 2015;1(1):37.

8. Healy GN, Dunstan DW, Salmon J, et al. Television time and continuous metabolic risk in physically active adults. Med Sci Sports Exerc. 2008;40(4):639-645.

9. Katzmarzyk PT, Church TS, Craig CL, Bouchard C. Sitting time and mortality from all causes, cardiovascular disease, and cancer. Med Sci Sports Exerc. 2009;41(5):998-1005.

10. Füzéki E, Engeroff T, Banzer W. Health benefits of light-intensity physical activity: a systematic review of accelerometer data of the National Health and Nutrition Examination Survey (NHANES). Sports Med. 2017;47(9):1769-1793.

11. Blodgett J, Theou O, Kirkland S, Andreou P, Rockwood K. The association between sedentary behaviour, moderate-vigorous physical activity and frailty in NHANES cohorts. Maturitas. 2015;80(2): 187-191.

12. Mekary RA, Willett WC, Hu FB, Ding EL. Isotemporal substitution paradigm for physical activity epidemiology and weight change. $A m J$ Epidemiol. 2009;170(4):519-527.

13. Tsoi KK, Chan JY, Hirai HW, Wong SY, Kwok TC. Cognitive tests to detect dementia: a systematic review and meta-analysis. JAMA Intern Med. 2015;175(9):1450-1458.

14. Giggins O, Clay I, Walsh L. Physical activity monitoring in patients with neurological disorders: a review of novel body-worn devices. Digit Biomark. 2017;1:14-42.

15. Shimada H, Makizako H, Doi T, et al. Combined prevalence of frailty and mild cognitive impairment in a population of elderly Japanese people. J Am Med Dir Assoc. 2013;14(7):518-524.
16. Lopopolo RB, Greco M, Sullivan D, Craik RL, Mangione KK. Effect of therapeutic exercise on gait speed in community-dwelling elderly people: a meta-analysis. Phys Ther. 2006;86(4):520-540.

17. Chen LK, Liu LK, Woo J, et al. Sarcopenia in Asia: consensus report of the Asian Working Group for Sarcopenia. J Am Med Dir Assoc. 2014; 15(2):95-101.

18. Fukutomi E, Okumiya K, Wada T, et al. Relationships between each category of 25-item frailty risk assessment (Kihon Checklist) and newly certified older adults under long-term care insurance: A 24-month follow-up study in a rural community in Japan. Geriatr Gerontol Int. 2015;15(7):864-871.

19. Kamada M, Shiroma EJ, Harris TB, Lee IM. Comparison of physical activity assessed using hip- and wrist-worn accelerometers. Gait Posture. 2016;44:23-28.

20. Mannini A, Intille SS, Rosenberger M, Sabatini AM, Haskell W. Activity recognition using a single accelerometer placed at the wrist or ankle. Med Sci Sports Exerc. 2013;45(11):2193-2203.

21. Pate RR, O'Neill JR, Lobelo F. The evolving definition of "sedentary". Exerc Sport Sci Rev. 2008;36(4):173-178.

22. Trost SG, Pate RR, Freedson PS, Sallis JF, Taylor WC. Using objective physical activity measures with youth: how many days of monitoring are needed? Med Sci Sports Exerc. 2000;32(2):426-431.

23. Nagai K, Miyamato T, Okamae A, et al. Physical activity combined with resistance training reduces symptoms of frailty in older adults: a randomized controlled trial. Arch Gerontol Geriatr. 2018;76:41-47.

24. Mekary RA, Lucas M, Pan A, et al. Isotemporal substitution analysis for physical activity, television watching, and risk of depression. Am J Epidemiol. 2013;178(3):474-483.

25. Powell KE, Paluch AE, Blair SN. Physical activity for health: What kind? How much? How intense? On top of what? Annu Rev Public Health. 2011;32:349-365.

26. Garber CE, Blissmer B, Deschenes MR, et al. American College of Sports Medicine position stand. Quantity and quality of exercise for developing and maintaining cardiorespiratory, musculoskeletal, and neuromotor fitness in apparently healthy adults: guidance for prescribing exercise. Med Sci Sports Exerc. 2011;43(7):1334-1359.

27. Sparling PB, Howard BJ, Dunstan DW, Owen N. Recommendations for physical activity in older adults. BMJ. 2015;350:h100.

28. Willey JZ, Moon YP, Kulick ER, et al. Physical inactivity predicts slow gait speed in an elderly multi-ethnic cohort study: The Northern Manhattan Study. Neuroepidemiology. 2017;49(1-2):24-30.

29. Warren JM, Ekelund U, Besson H, et al. Assessment of physical activity - a review of methodologies with reference to epidemiological research: a report of the exercise physiology section of the European Association of Cardiovascular Prevention and Rehabilitation. Eur J Cardiovasc Prev Rehabil. 2010;17(2):127-139.
Clinical Interventions in Aging

\section{Publish your work in this journal}

Clinical Interventions in Aging is an international, peer-reviewed journal focusing on evidence-based reports on the value or lack thereof of treatments intended to prevent or delay the onset of maladaptive correlates of aging in human beings. This journal is indexed on PubMed Central, MedLine,

\section{Dovepress}

CAS, Scopus and the Elsevier Bibliographic databases. The manuscript management system is completely online and includes a very quick and fair peer-review system, which is all easy to use. Visit http://www.dovepress. com/testimonials.php to read real quotes from published authors. 